\title{
GRADES: A New Instrument for Spectroscopy of the Local Hot Gas
}

\author{
M. Hurwitz ${ }^{1}$, C. McKee ${ }^{1}$, J. Edelstein ${ }^{1}$, J. Vallerga ${ }^{1}$, P. Jelinsky ${ }^{1}$, \\ M. Freyberg ${ }^{2}$, and D. Breitschwerdt ${ }^{2}$ \\ 1 Space Sciences Laboratory, University of California, Berkeley, California 94720- \\ 7450, U.S.A. \\ 2 Max-Planck-Institut für extraterrestrische Physik, D-85740 Garching, Germany
}

\begin{abstract}
Competing models for the origin of the local component of the diffuse $\mathrm{X}$-ray background span more than an order of magnitude in temperature. We propose to test these models with a new instrument, the GRating Array Diffuse EUV Spectrometer (GRADES), which is sensitive to diffuse emission in the $90-260 \AA$ bandpass, and offers a peak spectral resolution of about $\lambda / 127$.
\end{abstract}

\section{Introduction}

Million-degree gas is often referred to as "X-ray gas". This misnomer belies the uncomfortable reality that extrapolation of X-ray observational limits to the total plasma luminosity is critically dependent on the details of cooling processes that are not well understood. In collisional ionization equilibrium (CIE) near $10^{6} \mathrm{~K}$, the total plasma luminosity divided by the luminosity at wavelengths below $100 \AA$ is a factor of 5 . Million-degree gas is extreme ultraviolet or "EUV gas." Even after foreground absorption from the local cloud at about $2 \times 10^{18} \mathrm{~cm}^{-2}$ (Vallerga et al. 1993), the most energetically favorable band to study a CIE plasma at $10^{6} \mathrm{~K}$ is near $200 \AA$. The band contains strong emission lines from various ionization stages of iron; if the gas is in CIE, their ratios determine the temperature independent of abundance uncertainties. The strength of the Fe lines relative to those of low-depletion elements can determine the relative CIE abundances. If underionized nonequilibrium prevails (as in conduction [Slavin 1989]), the EUV may carry a higher fraction of the radiated power; line ratios can distinguish underionized emission from the CIE case. Because the absolute strength of the collisionally excited EUV emission lines depend sensitively on the electron temperature, uncharacteristically low values (e.g. overionized nonequilibrium) dramatically alter the EUV spectrum (Breitschwerdt and Schmutzler 1994). For reference: with a mean emission measure of $0.0038 \mathrm{~cm}^{-6} \mathrm{pc}$ (Snowden et al. 1997), and a temperature of $10^{6} \mathrm{~K}$, the strongest CIE emission line is expected to have a flux of about 120 photons $\mathrm{cm}^{-2} \mathrm{~s}^{-1} \mathrm{sr}^{-1}$ (hereafter Line Units or LU).

Observational limits have been set at the $1000 \mathrm{LU}$ and $500 \mathrm{LU}$ level (Labov and Bowyer 1991 and Smith et al. 1996, resp.). A deep spectrum taken with $E U V E$, the Extreme Ultraviolet Explorer (Bowyer 1995), suggests 
that the emission measure near $10^{6} \mathrm{~K}$ may be a factor of about four below the value from Snowden et al. (Jelinsky et al. 1993). This result, however, relies on subtraction of a very large background signal. Also using EUVE, Vallerga et al. find a few marginally significant detections in directions where the $1 / 4 \mathrm{keV}$ emission is bright. Spectrographs for diffuse emission between 350 and $1100 \AA$ have recently been developed (Bowyer et al. 1997). Observations have not begun (as of 1 June 1997). If the foreground neutral absorption is near or above $2 \times 10^{18} \mathrm{~cm}^{-2}$, the brightest CIE plasma emission features in the $350-912 \AA$ band will fall below the instrument sensitivity.

\section{The Proposed GRADES Instrument}

Consider first a single slit plus cylindrical diffraction grating placed at grazing incidence with respect to the central ray (hereafter "channel"). Baffles restrict the field of view (FOV) to a cone about $10^{\circ}$ in diameter. Diffuse sky glow within the FOV will strike the grating and be focused within the plane of dispersion onto a planar photon-counting microchannel plate detector. We vary the spacing of the grooves to provide aberration control and flatten the focal surface (Harada et al. 1991). GRADES comprises 16 spectrograph channels, illustrated within the payload envelope of the Spartan Lite (a potential spacecraft carrier) in Figure 1. The off-axis channels are identical to the central one save for a rotation about an axis passing through the detector surface. Outside the entrance slit, each off-axis channel carries a small pick-off mirror to coalign the FOV with that of the central channel within the dispersion plane. Perpendicular to that plane, the FOV is about $20^{\circ}$. GRADES provides no angular resolution within its FOV. The integrated flux from point sources is far below the expected diffuse flux in this band. The continuum from the brightest white dwarf in the sky (HZ 43) is comparable to the detector background. The well known flare star AU Mic in outburst (Katsova et al. 1996) produces lines corresponding to $<0.5 \mathrm{LU}$ in diffuse flux when observed with GRADES. We summarize the top-level parameters in Table 1.

Without filters, scattering of bright emission lines of geocoronal and interplanetary origin (especially Lyman $\alpha$, with a typical flux of $\mathbf{3 5 0 0}$ Rayleighs [Chakrabarti et al. 1984]) would exceed the detector background. Page limits do not permit a full discussion of our rationale for filter material selection. The filter for $210-260 \AA$ will be $1500 \AA$ thick Aluminum; for $90-150 \AA$, the filter will be $1000 \AA$ thick Lexan plus $300 \AA$ of Boron. Intermediate wavelengths will be spanned by both materials, each covering half of the detector area. We show the system resolution and $2 \sigma$ sensitivity curve for an integration time of 26,000 seconds ( 1 day at $30 \%$ observing efficiency) in Figure 2. 

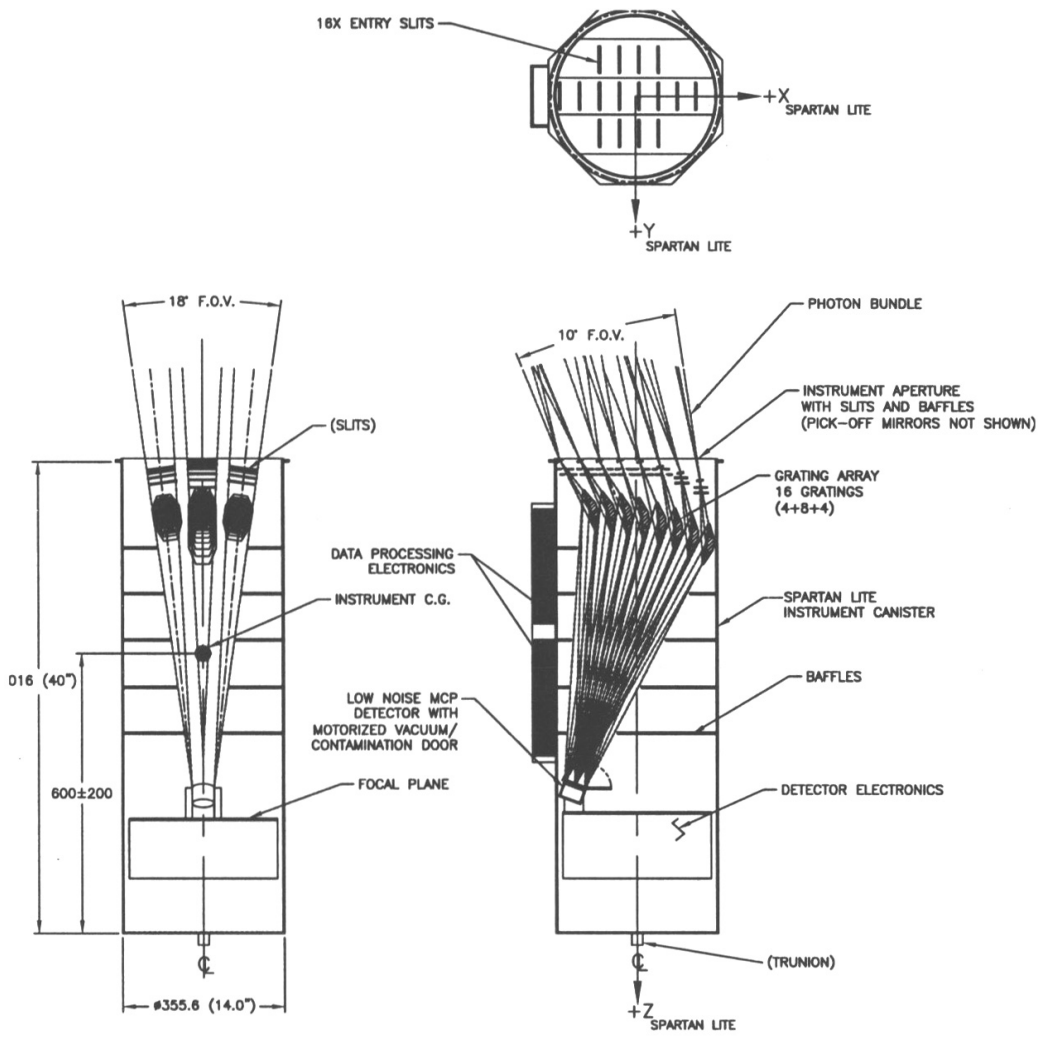

Fig. 1. Full 16-channel GRADES illustrated in Spartan Lite envelope.
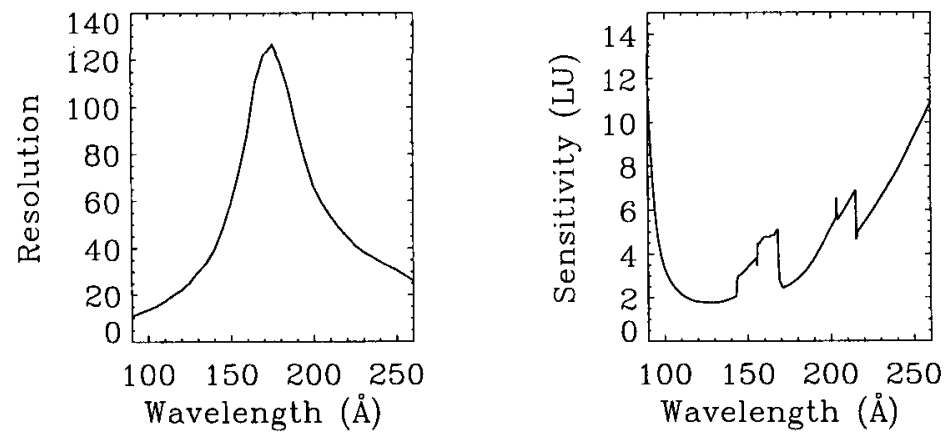

Fig. 2. GRADES spectral resolution $\lambda / \delta \lambda$ ( $76 \%$ energy width) and $2 \sigma$ sensitivity to emission lines (LU) for 1 day in low earth orbit at $30 \%$ observing efficiency. Discontinuities are associated with changes in instrument throughput caused by filter support bars or filter transmission edges. 
Table 1. Top-Level GRADES Parameters

\begin{tabular}{|l|l|}
\hline Entrance slits & $0.013 \times 6 \mathrm{~cm}$ \\
\hline Grating graze, blaze angle & $14^{\circ}, 2.6^{\circ}$ \\
\hline Groove density & $1330-1800 \mathrm{~mm}^{-1}$ \\
\hline Grating dimensions, $\mathrm{R}_{\text {curve }}$ & $9 \times 6 \mathrm{~cm}, 96 \mathrm{~cm}$ \\
\hline Coatings, Photocathode & Rhodium, NaBr \\
\hline Detector area, resolution & $5.5 \times 6 \mathrm{~cm}, 400 \times 400$ resels \\
\hline Filters & $1000 \AA \mathrm{Lex}+300 \AA$ Boron, $1500 \AA$ Alum. \\
\hline Field of View, Bandpass & $10 \times 20^{\circ}, 90-260 \AA$ \\
\hline Spectral Resolution $(76 \% \mathrm{EW})$ & $11-127(\lambda / \delta \lambda)$ \\
\hline $\mathrm{A}_{e f f} \times \Omega$ & $2.5 \times 10^{-4}-1.6 \times 10^{-3} \mathrm{~cm}^{2} \mathrm{ster}$ \\
\hline $2 \sigma \mathrm{Min}$. Detectable Line Flux & $1.8-12 \mathrm{LU} \mathrm{in} 26,000 \mathrm{sec}$ \\
\hline
\end{tabular}

\section{Conclusions}

Despite the importance of spectral diagnostics in the $\sim 200 \AA$ band, observations of the local diffuse plasma at these wavelengths are extraordinarily scant. A new instrument GRADES, optimized for spectroscopy of the diffuse emission from $90-260 \AA$, will permit the first true emission line diagnostics. Data from GRADES will enable us to constrain the plasma temperature, emission measure, gas phase abundance of iron, and two classes of nonequilibrium effects independently.

\section{References}

Bowyer, S. (1995): in "X-ray and EUV/FUV Spectroscopy and Polarimetry", ed. S. Fineschi, Proc. SPIE 2517, 97

Bowyer, S., Edelstein, J., Lampton, M. (1997): accepted for ApJ

Breitschwerdt, D., Schmutzler, T. (1994): Nature 371, 774

Chakrabarti, S., Kimble, R., Bowyer, S. (1984): JGR 89, No. A7, 5660

Harada, T., Kita, T., Bowyer, S., Hurwitz, M. (1991): in "International Conference on the Application and Theory of Periodic Structures", ed. J. M. Lerner \& W. R. McKinney, Proc. SPIE 1545, 2

Jelinsky, P., Vallerga, J., Edelstein, J. (1993): ApJ Lett 417, L41

Katsova, M., Drake, J., Livshits, M. (1996): in "Astrophysics in the EUV", ed. S. Bowyer and R. F. Malina, Kluwer Academic, Dordrecht, 175

Labov, S., Bowyer, S. (1991): ApJ 371, 810

Slavin, J. (1989): ApJ 346, 718

Smith, B., Pfafman, T., Bloch, J., Edwards, B. (1996): in "Astrophysics in the EUV", ed. S. Bowyer and R. F. Malina, Kluwer Academic, Dordrecht, 283

Snowden, S., Egger, R., Finkbeiner, D., Freyberg, M., Plucinsky, P. (1997): submitted to $A p J$

Vallerga, J., Vedder, P., Craig, N., Welsh, B. (1993): ApJ 411, 729 